\title{
Téoros
}

Revue de recherche en tourisme

\section{Nouvelles réflexions sur le taux de fonction touristique}

\section{Pierre Defert}

Volume 7, numéro 3, novembre 1988

Économie du tourisme

URI : https://id.erudit.org/iderudit/1080381ar

DOI : https://doi.org/10.7202/1080381ar

Aller au sommaire du numéro

Éditeur(s)

Université du Québec à Montréal

ISSN

0712-8657 (imprimé)

1923-2705 (numérique)

Découvrir la revue

Citer cet article

Defert, P. (1988). Nouvelles réflexions sur le taux de fonction touristique.

Téoros, 7(3), 24-28. https://doi.org/10.7202/1080381ar d'utilisation que vous pouvez consulter en ligne.

https://apropos.erudit.org/fr/usagers/politique-dutilisation/ 
Lorsqu'en 1643, le physicien italien Torricelli inventa le baromètre, combien de temps $s^{*} e ́ c c u l a-t-i l$ avant que l'usage de cet appareil fut généralisé pour une meilleure connaissance de l'atmosphère et la prévision du temps? Nous n'en savons rien mais toutes nos maisons ont aujourd'hui un barometre. Le retard dans l'emploi d'une valeur de mesure ainsi constatée par les sciences physiques se retrouve pareillement dans les sciences humaines. Car c'est en 1950 que nous avons imaginé une mesure permettant de mettre en relief l'importance du tourisme en un lieu donné: le taux de foncrion touristique (Tf $(\mathrm{t})$ ).

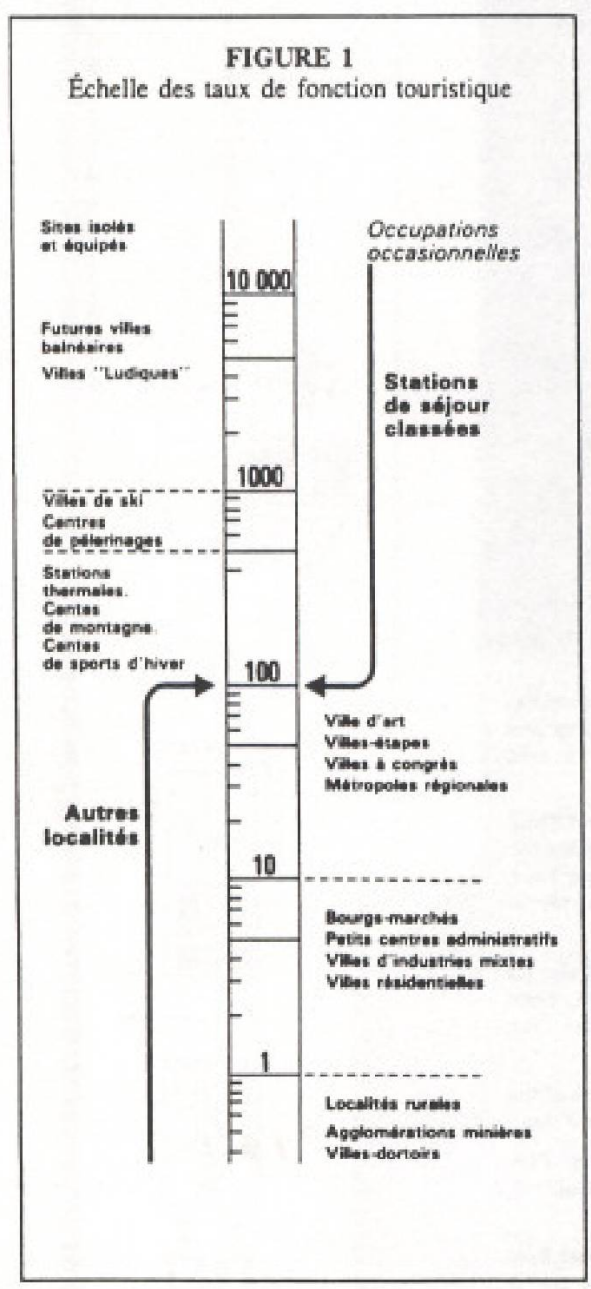

- Monsieur Pierre Defert est Recteur a I'Universite Berenice a Paris.
Près de 40 ans ont passé et il est urgent d'examiner si cette mesure présente toujours un intérêtt pour la connaissance du phénomène, si elle a été connue et appliquée par des observatoires d'économie touristique ou des bureaux de planification en dehors de l"usage que nous en avons fait nous-mémes dans de multiples études régionales.

\section{Définition du taux}

Rappelons d'abord les bases de ce calcul (nous renvoyons pour le détail de cette notion au cahier série C, No 5, publié en 1967 de l'excellente collection du Centre des Hautes Études Touristiques d'Aix-en-Provence, France $\left.^{(1)}\right)$ :

"Nous définirons le taux de fonction touristique comme le rapport du nombre de places $\mathrm{L}$, disponibles pour les hôtes dans la localité, multiplié par 100 , à la population permanente $\mathrm{P}$ de cette localité. Il s'écrit:

$\operatorname{Tf}(t)=\frac{L \times 100}{P}$

Le terme de "fonction touristique" est pris ici dans son sens économique et non dans son sens algébrique; en effet, il n'est pas question de relier par une formule mathematique la population au nombre de touristes immigrés en saison. Nous verrons toutefois qu'il peut exister des liaisons nuancées entre ces deux variables qui, dans certains cas, peuvent être dépendantes. Le terme de fonction touristique est donc pris dans le sens brut d'activité touristique de la localité.

La fonction est en fait un rapport entre deux éléments sociaux et les variations de chacun $\mathrm{d}^{+}$eux sont à priori indépendantes. Par suite, ce rapport ne peut faire l'objet d'une formulation algébriquel? ${ }^{27}$. Mieux encore, le terme fonction est ici emprunté au wocabulaire physiologique et assimile la ville à un organe. Son créateur est le géographe allemand Ratzel (1901). On reconnait comme foncrion urbaine les activités qui justifient l'existence de cet organe. et son développement. qui apportent les ressources nécessaires à sa vie (J. Beaujeu-Garnier et G. Chabot, op. cit. p. 105), à l'exclusion des activités entre habitants (city serving production). Dans le cas de la fonction touristique, tout se passe comme si le trafic externe (services et biens foumis aux touristes) se superposait au trafic interne par suite de l'installation intramuros de consommateurs venus de l'extérieur.
Les dénombrements, nombre de places et populations peuvent aussi être faits à l'échelon d'une région ou d'un État. On a ainsi un Tf (t) regional ou un Tf (t) national pour permettre des comparaisons.

\section{La méthode potentielle}

Le total des places disponibles dénombrées dans toutes les localités nous permet d'esti= mer la capacité potentielle de réception. Nous devons ouvrir ici une parenthèse au sujet de cette méthode, car on nous objectera que la capacité de réception, notion répondant à des fins theoriques, peut fort bien ne pas correspondre à la fréquentation. En fait, notre hypothèse est que cente capacité théorique de réception, si elle se fonde sur le réel, mesure au moins une fréquentation moyenne attendue. Certes, selon la conjoncture, un certain nombre de lits resteront éventuellement inoccupés, comme l'on pourra, inversement, atteindre la surcharge et obliger les automobilistes à coucher dans leurs voitures. Dans l'ensemble, toutefois, nous étudions ici des structures et non des mouvements.

I en résulte que les equipements mis en place pour recevoir les hótes éventuels, sous quelque forme d'hébergement que ce soit, traduisent la réalité économique profonde. On peut en tenir compte et les considérer comme exprimant l'importance de la population saisonnière qui fréquente la station. En fait, en pleine saison (au mois d'août par exemple) il est fort probable qu'ils fonctionnent à plein. II serait de toute manière possible de corriger cette approximation en déterminant un tawx moyen mensuel d'ouverture touristique, facteur dont nous possédons assez bien les valeurs, pour les hôtels, tant en France qu'en Suisse. La capacité de réception touristique de l'hơtellerie souffre de réductions mensuelles provenant soit de la fermeture saisonnière de certains hôtels, soit de la réfection de parties d"hötels dont les chambres ne sont plus alors présentables à la clientèle $e^{(3)}$.

\section{Le contact: visiteurs/visités}

1) Une précision s'impose à l'esprit. On parle beaucoup pour exprimer l'essence du tourisme d'une fonction d'accueil ou d'hospitalité. En réalité, le Tf (i) représente un contact humain lequel repose sur deux elements:

- une base fixe (l'enclume): la masse des hôtes qui accueilleront les voyageurs:

- un élément mobile (le marteau): les étrangers venus dans la région. 
La langue française confond sous le mème rerme $d$ hötes les deux groupes. On lui préferera la langue anglaise qui distingue "host" et "guest".

Cette fonction de contact est d'une durée limitéc puisqu'il n'y a pas installation permanente. Cette durée limitée s'appelle la saison. Elle s'exerce en un point localement bien déterminé: la station. Le choix des points les meilleurs relève d'une méthode de recherche que nous avons développée par ailleurs: la localisation ${ }^{(4)}$.

2) Il est apparu chez divers auteurs assez fréquemment une autre notion, celui d'intensite touristique (Samolewitz, 1950). L'analogie que l'on peut faire entre un courant de tourisme et un courant électrique est assez maladroite car la venue sous un certain volume de visiteurs étrangers en contact avec les résidents locaux peut créer des relations diverses:

a) Indifférence lorsque l'écart des cultures est tel que la juxtaposition des premiers et des seconds ne laisse pas apparaĭtre de réactions favorables ou hostiles en raison même du peu de surface sociale qui se trouve en contact depart et d'autre (surface de frottement). Cette indifférence peut ètre voulue ou méme provoquée par un isolement matériel (enceinte d'un village de vacances, $d^{\text {tun }}$ terrain de camping, jardins disposés comme des glacis autour d'un hôtel de luxe laissant à l'écart de toute approche la population autochtone. Seuls les taxis ou les conducteurs de bus sont autorisés à passer la barrière d'un vrai ghetto.

b) Hostilite latente mais ne se traduisant pas par des rejets. Elle s'exprime surtout par le relais du personnel de service qui a le contact avec les touristes (serveurs, bagagistes, réceptionnistes, animateurs) et qui peut faire connaître à leurs hôtes l'opinion générale de la population environnante qui vit à l'écart de l'activité touristique sans y participer. Les voyageurs étrangers sont plus ou moins désirables selon leurs nationalités et selon les comportements qu'ils manifestent en public.

c) Une acculturation qui se produit largement dans un contact atomisé entre des loueurs de résidences meublées et leurs clients. Ou bien entre des groupes de jeunes invités à participer à des tăches d'assistance, de connaissance ou de perfectionnement linguistique. L'installation de souks temporaires un jour de la semaine dans le hall d'un palace ressort de ce contact puisqu il permet aux étrangers d'apprécier puis d'acheter sans fatigue des produits de l'artisanat local. La réception organisée dans des familles autochtones de touristes étrangers (Scandinavie, USA) va dans ce sens et cente tendance est bien perçue.

d) On peut noter enfin un comportement strictement mercantile qui fut celui des populations d'accueil a l'aube du tourisme dans. des régions pauvres. Une mendicité spontanée et chronique cherche à obtenir le maxi- mum de cadeaux, de dons des étrangers. Ils sont taxés et surtaxés au prix fort dans leur méconnaissance d'une monnaie locale et des prix locaux (Italie du sud, Orient).

À la limite, le vol ou le chapardage de leurs équipements (photo) ou de leurs devises est considéré comme une rançon logiquement perçue sur ces visiteurs. Comme le pillage des épaves attirées par les naufrageurs d'autrefois sur les brisants où les cargaisons étaient réparties entre participants.

Toutes les réactions de contact sont d'ordre qualitatif et peuvent toutes se référer à un même $\mathrm{Tf}(\mathrm{t})$ qui est strictement quantitatif. Ainsi se marque la limite de signification du taux de fonction touristique.

\section{Le tertiaire n'est plus significatif}

Nous avions pensé caractériser autrefois, à la suite de certains auteurs suisses, la fonction touristique par une équation de services, notamment commerciale. On pensait qu"à partir de $60 \%$ de travailleurs employés dans les commerces et services urbains nous pouvions détecter une fonction touristique. Cette proposition ne peut plus être acceptée car en un demi-siècle (plutôt moins dans les pays développés), la diminution du travail humain dans l'industrie lourde et l'agriculture fait que des services sont en place pour accroiltre la productivité de ces deux secteurs sans nécessairement qu'il s'agisse de services touristiques. On peut concevoir des villes dont l'activité est purement tertiaire et qui n'ont qu'un rôle très faible sinon nul dans le tourisme. L'âge de l'informatique a accru leur importance car elles sont désormais faites de réseaux bancaires, financiers, administratifs, commerciaux, médiatiques appliqués à des domaines qui ne sont pas des loisirs.

Dans une station de tourisme, la capacité d'accueil varie fréquemment au cours de l'année. Certains hốtels dits "saisonniers" sont ouverts seulement en été, ou en été et en hiver et fermes au printemps et en automne. La capacité d'un terrain de camping quand la vie de plein air est impossible, ne signifie rien. Beaucoup de meublés n"ont pas de chauffage.

En somme, un calcul scrupuleux du Tf (t) conduirait à estimer d'un mois sur l'autre ou d'une saison sur l'autre, le potentiel d'aceueil et de le rapporter ensuite a la population permanente, laquelle n'a pas les mémes raisons de varier et présente une garantie de stabilité (sauf population de certains établissements scolaires, hospitaliers, maisons de retraite, collectivités religieuses, casernes, etc.). On est conduit ainsi à considérer que le taux le plus significatif est celui de la pleine saison où le potentiel d'accueil est utilisé à $100 \%$ mais si on doit faire une étude saisonnière de l'activité touristique, en particulier dans le but d"etaler la saison, il faudrait tenir compte de takx rouristiques saisonniers représentés sur un graphe. Ils auraient le mérite de permettre des comparaisons entre stations diverses. Nous n'avons trouvé à notre connaissance aucune application de taux saisonniers dans les études qui nous sont parvenues.

\section{Actualité de la formulation}

La première application de cette formule, imaginée par nous en 1950 , a été faite en septembre 1957 dans notre rapport sur le développement touristique de la région de Gruyère en Suisse. Une dizaine de chercheurs et étudiants l'ont utilisés depuis dans divers travaux. La fonction touristique d"une localité (que nous caractérisons done par le rapport autochtones-immigrés saisonniers) n'a pas jusqu'ici été explicitée par les géographes, urbanistes, sociologues qui ont étudié le phénomène urbain. En effet, les trois définitions de F. Juillard (ville rentière du sol, ville insulaire, ville urbanisante) peuvent s'appliquer à la ville de Tourisme. Le taux de fonction touristique que nous proposons est aujourd hui sur le point de trouver (à posteriori) une consécration administrative. Un projet de texte en cours d'étude pour le classement des stations de tourisme introduit cette notion nouvelle (dans l'esprit du législateur, mais habituelle aux geographes et urbanistes) de la juxtaposition temporaire de deux masses humaines. Ce projet réserve la qualité de station aux localités offrant en saison une capacité d'hébergement égale ou supérieure à $35 \%$ du chiffre de la population sédentaire (les terrains de camping étant exclus, on ne voit pas à quel titre). C'est dire, en somme, qu"un taux de fonction touristique de:

$$
\frac{35 \times 100}{100}=35
$$

définirait le seuil légal de la vie touristique. Ce projet reconnait par là-mème implicitement la valeur de la méthode de la capacité potentielle que nous avons développée.

D'autre part, une jurisprudence établie par le Conseil Supérieur du tourisme en France et suivie par le Conseil d'État tend à considérer pareillement le volume de la population flottante accueillie en saison.

Son estimation part Egalement de la méthode de la capacité potentielle et repose sur le nombre de lits en hôtels de tourisme. Le critère admis exige une capacité d'hébergement égale à $40 \%$ de la population sédentaire de la commune, fraction ou groupe de communes à classer ${ }^{(\$)}$. Ce Tf (t) égal à 40 est en réalité un taux de fonction hórelière réduit. Son inadéquation est notoire puisqu'il néglige la présence d'hôtes utilisant d'autres formes d'hébergement (l'hébergement hơtelier représentant souvent moins de $20 \%$ de la capacité d'accueil globale d'une station).

En résumé, si le Tf (t) est supérieur à 100, les visiteurs sont dominants et imposent leurs habitudes et leurs cultures à leurs hótes. Ils expriment sans réserve leur droit à la différence. 
Si le Tf (c) est inférieur à 100 , le phénomène inverse se produit. Ce sont les visiteurs qui se soumettent à leurs hôtes et font un effort pour assimiler leur culture.

\section{L'échelle des taux. Critique et cas limites}

Calculés sur un grand nombre de localités, les $\operatorname{Tf}(\mathrm{t})$ présentent un éventail de variation assez grand. Les cas-limites sur lesquels nous reviendront, sont:

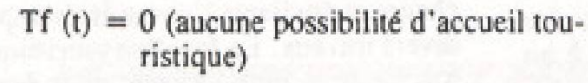

et

Tf $(t)=\infty$ (il $n^{\prime \prime} y$ a que des touristes, la population résidant en permanence est inexistante ou se réduit à quelques personnes: gardiennage, services d'entretien, etc.). Dans cet intervalle, des seuils sont à parquer (figure 1).

\section{Le tourisme des îles: le risque d'ethnolyse}

L'evolution historique montre que les grandes îles semi-continentales ont été assez tôt des États autonomes (Australie, NouvelleZulande, Irlande). Cette conscience a été vite acquise aussi pour des territoires aussi différents que le Groẻnland, Madagascar, Bornéo. Nous ne sommes pas là à la dimension insulaire telle que nous l'avons définie plus haut. Pour les îles, d'une superficie inférieure à $10000 \mathrm{~km}$, la tentation de tirer parti du tourisme est certaine, puisqu'il s'agit d'une fessource nouvelle.

Toutefois, l'ambiguité reste grande et repose sur deux évolutions difficiles à contrôler: $S$ 'agit-il de troquer un colonialisme d'autrefois contre un néo-colonialisme contemporain? En effet, la mainmise du marché par les biais des tours operateurs investisseurs et des organismes bancaires qui peuvent assurér le développement fait que le tourisme fait perdre le contrôle du tourisme par l'ile ellemẻme. Ce mécanisme d'aliénation économique, très classique (Antilles, Canaries, Polynésie) a été maintes fois décrit. Le marché est controblé par des forces extérieures renforcées par les réseaux de transport (charters) qui apportent la clientèle. La négocia. tion est un rapport de forces et, la concurrence aidant, l'exportation du tourisme n'échappe guère aux lois d'airain qui ont depuis toujours contrōlé le marché des matières premières du Tiers-Monde, végétales (coton, cacao, café) ou minérales (métaux non ferreux, pétrole).

L'observation des effets du tourisme récent dans certaines illes du Pacifique en est une illustration. Les premiers groupes adoptent les vétements (paréos), la nourriture, I'habitat (faré) des insulaires polynésiens. Ce deguisement constitue un rite de l'accueil et ouvre la porte de l'hospitalité. Le touriste découvre plus ou moins un genre de vie qui le séduit par son exotisme et son étrangeté et il essaie de s"y conformer pour quelques semaines. Son impact (quelques dizaines d'individus) reste minoritaire face aux centaines d'indigènes qui ne se sentent pas pour autant évincés de leurs territoires ou recolonialisés sous une autre façon.

En revanche, quand des charters de plusieurs centaines de places, déversent sur les iles Canaries ou à Madère des milliers de touristes occidentaux installés dans des constructions neuves créées pour eux, dotées de tout le confort, face à de nouvelles plages, équipées de boutiques et de supermarchés abondamment pourvus de produits occidentaux, ce sont les indigènes qui font un effort de novation pour adopter les costumes, la nourriture, les modes de vie et le parler de leurs visiteurs. $\grave{A}$ la limite, la culture autochtone peut être complètement effacée (ethnolyse) car les touristes s'en soucient peu et constituent une véritable implantation ethnique, volontaire et dominatrice.

On peut également reprendre cet aspect des choses en notant l'apparition d'une "conscience de classe" touristique. En voici le contenu:

Le touriste se définit par sa conscience de classe. Celle-ci se fait jour quand le touriste est en nombre suffisant pour sentir que son espèce forme un groupe au sein du milieu qui la reçoit. Il impose par suite à ce milieu ses besoins particuliers, ses gouts, ses habitudes. D'où des conflits possibles. Quand les responsables locaux admettent la nécessité de satisfaire les besoins (urbains) économiques et culturels du groupe de touristes, ils découvrent la notion de station de tourisme: place, lieu ou un ensemble d'immigrés est assez fort pour se faire reconnaitre comme tel et refuser l'assimilation autochtone. La reconnaissance des besoins du groupe temporaire entraine les investissements, l'équipement, etc.

La station de tourisme n'a done pas à priori une définition économique: elle est un fait sociologique de classe. Ce sont les immigrés. saisonniers qui la créent en manifestant leur personnalite et leur présence et en choisissant de se distinguer des habitants permanents (voire de les scandaliser). On est donc ici très loin des chances d'acculturation puisque les deux groupes d'hótes s'affrontent volontairement en tant que tels. Un affrontement d'intérêts économiques (clients) remplace l'hospitalité et l'essai de compréhension des problèmes. Le bilan humain est nul ou négatif.

Un cas intéressant à discuter est un caslimite, celui d'une installation touristique dans une zone sans résidents permanents antérieurs. Disons que ce cas de figure ne peut se trouver dans les pays développés où le sol est occupé de longue date par l'agriculture ou la forêt. Mais certains sites de montagne, en altitude glaciaire. offrent ce caractère et ont fait l'objet d'installations de sports d'hiver. Le cas est plus fréquent dans les pays du Tiers-Monde sur des littoraux des îles désertiques où un promoteur installe soit un village de vacances, soit un ensemble hôtelier de grande classe. Dans ce cas, la theorie voudrait qu'on puisse parler d'un Tf (t) tendant vers l'infini puisque le numéra= teur de notre fraction peut atteindre une grande différence avec le dénominateur.

Mais ce dénominateur peut-il être nul? Erreur de le croire. On peut citer pourtant la randonnée en car-camping dans le Nord canadien ou la traversée du Sahara. Mais le plus souvent, la résidence fixe oblige à la permanence de services et d'employés qui sont nécessaires au bon fonctionnement de l'installation. Un grand hôtel aura dans sa galerie une agence bancaire, une agence de voyages, un bureau postal, quelques marchands de souvenirs, au besoin un lieu de culte. Le tout employant quelques autochtones. Le personnel de service, souvent une personne par touriste dans une catégorie luxe, s'il n'est pas logé sur place peut l'ètre à proximité. Si la station a quelque ancienneté, elle aura attiré autour de ses hôtels ou de ses résidences toute la hiérarchie des commerces et des services, pouvant aller de l'indispensable (épicerie) au superflu (fleuriste). On pense qu'en général 10 touristes requièrent la présence perma= nente d'un employé dans leurs besoins. Ce rapport constitue done un seuil au-dessus duquel le $\mathrm{Tf}(\mathrm{t})$ ne peut pas monter. Il est évidemment variable avec le genre de tourisme pratiqué, la qualité des hótes, la saison de fréquentation, etc. Sa valeur est:

$\operatorname{Tf}(t)=L \times 100 \times \frac{10}{L}=1000$

Ce n'est donc pas l'infini.

\section{Application au tourisme insulaire}

L'examen du taux de fonction touristique que nous avons pu établir sur une cinquantaine d'iles, connaissant avec une bonne approximation leur population et leur capacité de réception (hótellerie et para-hótellerie) cela nous permet quelques remarques:

Nous avons regroupé ces taux par ensembles insulaires en donnant une moyenne:

- Méditerranée:

- Atlantique/Caraibes:

13,52

- Pacifique:

- Océan Indien:

Grosso modo, cela représente assez bien 4 stades de développement touritique au niveau insulaire.

\section{1 - La Méditerranée $(13,52)$}

Le taux est dans l'ensemble supérieur à 10. ce qui signifie en clair qu'on dénombre plus de 10 étrangers visiteurs pour 100 habitants.

Nous avons là des îles très développées comme la Corse, les Balćares, Rhodes. Djerba, la Sicile, etc. 


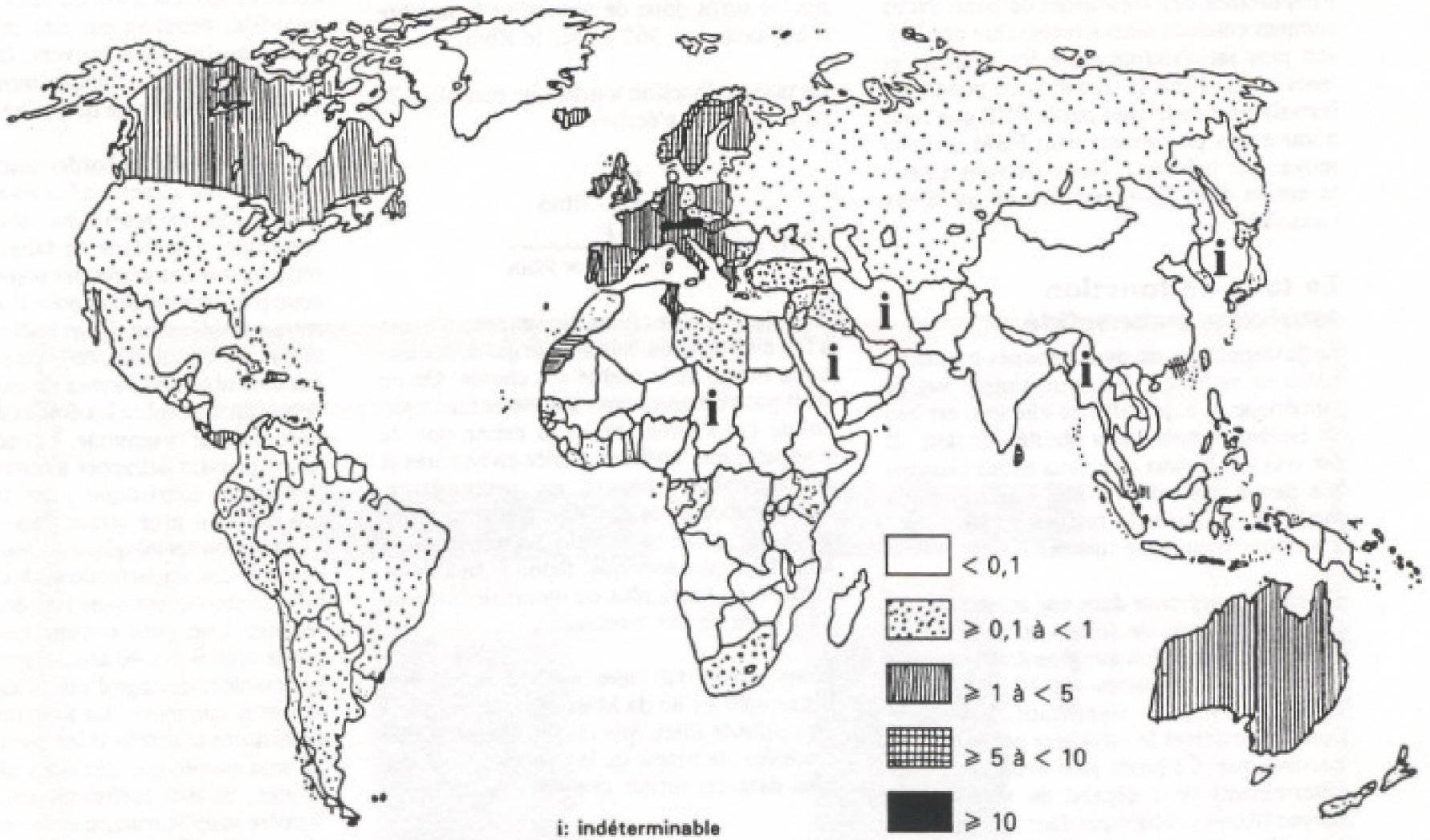

Le taux de fonction touristique est le rapport qui existe entre la capacité d'accueil (nombre de lits en hótels principalement) et la population selon la relation Tf(t) = NL sur $\mathrm{P} \times 100$. Calculé au niveau des pays, ce taux permet d'apprécier le degré de développement touristique et il est plus facile à connaitre que le nombre de visiteurs ou le nombre de nuitées que les services statistiques des différents pays ne recensent pas toujours avec exactitude.

Il s'agit avant tout de flux de vacanciers européens se rendant dans des territoires européens (sauf Djerba). L'affrontement ethnique ou racial est peu marqué. Il est pratiquement inexistant dans le cas d'iles fréquentées. par des nationaux du mẻme pays (Suédois à Gotland, Anglais dans l'île de Man ou à Jersey). Ces îles ont fait dans l'ensemble objet de nombreuses études géographiques ou économiques. Ce qui ne veut pas dire que le problème de la capacité de charge ne se pose pas et ne mériterait pas de trouver des solutions à certaines époques de l'année.

\section{2 - Atlantique/Caraïbes $(6,99)$}

Le marché américain joue un rôle fondamental pour le développement de ce secteur. Les taux ont tendance à augmenter vite (Caìmans: 64). Le tourisme se substitue a la monoculture de l'exportation de la canne à sucre (la Barbade). L'affrontement ethnique existe mais il est fortement atténué par la prédominance du mode de vie américain et le règne du dollar. Pour de nombreuses iles, le tourisme est un espoir bien fondé et les liaisons avec les zones-marchés sont courtes et rapides.

\section{3 - Le Pacifique $(4,38)$}

Ce taux est relativement faible mais en raison de la situation démographique, le dan- ger de surcharge est récl en cas de doublement de la fréquentation. Les effets peuvent être destructeurs à Guam, Tahiti, Polynésié française, Tonga, Samoa. Une protection de l'environnement est plus que jamais nécessaire pour sauvegarder les aspects naturels (ile de Păques, Galapagos, atolls, volcans). L'objectif de Tahiti, de couvrir au moins $35 \%$ de ses importations par des recettes du tourisme parait ambitieux.

\section{4 - Océan Indien $(1,06)$}

Il n'existe pas de risques immédiats de destructuration sauf aux Seychelles. Si les taux venaient à croítre pour atteindre des valeurs entre 5 et 10 , il y aurait lieu de surveiller la fragilité des populations et des sites qui ne pourraient pas résister à un impact de courants occidentaux.

Ces considérations sur les taux de fonction touristique seront à recouper avec les analyses que nous donnons plus loin en fonction d'autres facteurs conomiques.

\section{Le Tf (t) au niveau mondial}

La représentation cartographique ne peut êture que très grossière, en effet les petits pays de moins de $50000 \mathrm{~km}^{2}$ ainsi que les iles ne peuvent ètre convenablement représentés.
Certains jouent pourtant un rôle touristique déterminant (Chypre, Madère, Hong Kong, Jamaïque, Saint-Marin, Andorré, etc.).

On remarque que les pays européens viennent en tête. Ils absorbent en effet plus de $75 \%$ des courants internationaux de tourisme avec des recettes correspondantes. La densité de population y est moyenne sans atteindre certaines valeurs de pays asiatiques. La Suisse, I'Autriche et les pays de la bordure méditerranéenne marquent ici leur importance.

La faiblesse des destinations exoriques à la mode avec un taux inférieur à 1 est patente malgré l'intérết publicitaire qu'ils ont dans les catalogues des voyagistes. Par exemple, Mexique, USA, Pérou, URSS, Kénya, Japon. Il y a un décalage entre l'image touristique publicitaire et la fonction réelle d'accueil. Dans les pays du Tiers-Monde à faible densité de population, cet écart augmente artificiellement le taux.

La faiblesse est encore plus grande pour de grands espaces faiblement tourististifiés comme la Chine, I'Afrique centrale, le Brésil. Le taux est inférieur à 0,1 . Il y a une carence d'équipements d'hébergement provenant de la faiblesse des échanges intérieurs. 
Ces remarques nous confirment une liaison entre la fonction touristique et le degré de développement économique quelle que soit l'importance des ressources de base. Nous sommes conduits ainsi à rechercher une liaison plus satisfaisante entre les visiteurs et leurs hôtes. Nous la trouvons en pondérant les valeurs numeriques par le $\mathrm{PNB}$ que nous nommerons plus exactement RBM (revenu moyen par habitant). Nous arrivons ainsi a la notion d'un If ( $t)$ pondére ou au mieux consolide.

\section{Le taux de fonction touristique consolidé}

La juxtaposition de deux groupes humains, hôtes et visiteurs, par leur simple valeur numérique, si expressive qu'elle soit, est loin de rendre compte de la réalité des faits. Il est vrai que le taux que nous avons imaginé n'a pas la prétention d'être une valeur de synthèse, reflétant la totalité du tourisme. Pourrions-nous faire mieux?

En effet, la présence dans une station de 100 touristes porteurs de dollars ou de marks, à côtué d'une population indigène dont le revenu en pesos ou en sucres est 10 fois moins important est aussi significatif. Il importe donc de pondérer le voyageur par son poids cconomique. Ce poids peut avoir des effets déterminants et il dépend du revenu brut moyen (Rbm) par habitant dans le pays émetteur. Nous pouvons le comparer (en dollars) à ce mème revenu souvent fort bas dans le pays récepteur.

Pour ce faire, le nombre potentiel de places nous importe moins qu'une statistique cette fois nécessaire des mouvements de tourisme. Il faut avoir un décompte assez précis des nuitées par nationalités d'arrivants. Dans l'ensemble, les ministères de Tourisme, soucieux de comptabilité touristique, tiennent assez volontiers compte de cette différenciation.

En somme, c"est l'écart de niveau de vie entre les arrivants et la population d'accueil qu'il faut introduire dans notre modèle.

\section{Mode de calcul}

Soit $\mathbf{N}$ le nombre de nuitées totales avec

$\mathbb{N}=n_{1}+n_{2}+n_{3} \ldots+n_{x}$, pour $X$ nationalites différentes recensées parmi les touristes

Nous chercherons Egalement les valeurs du Rbm/hab pour chaque nationalité, soit $\mathrm{Rbm}_{1}, \mathrm{Rbm}_{2}, \mathrm{Rbm}_{3} \ldots \mathrm{Rbm}_{\mathrm{x}}$.

Chaque nombre de nuitées sera pondéré par Rbm correspondant. $n_{1} \times$ Rbm $_{1}, n_{2} \times$ $\operatorname{Rbm}_{2} \ldots \bar{\pi}_{X} \operatorname{Rbm}_{x}$

La somme en valeurs s.exprime ainsi:

$$
\sum_{i=1}^{n} R b m
$$

La population indigène doit être considérée aussi sous l'angle des nuitées et du revenu pour que les termes du taux soient homogènes. Il suffit done de multiplier le nombre d'habitants par 365 et par le Rbm.

Le taux de fonction touristique consolidé Tf (t) cons. peut s'écrire:

Tf (t) cons. $=\frac{\mathrm{i}_{=}^{\frac{\mathrm{n}}{\Sigma}} \mathrm{Rbm}}{\mathrm{P} \times 365 \times \mathrm{Rbm}}$

Il va de soi qu"on obtient une expression tout à fait différente du taux simple qui donne une autre image de la réalité des choses. On ne peut pas comparer taux simple et taux consolidé car l'erreur serait la même que de comparer une surface calculée en hectares et un périmètre mesuré en hectomètres. L'application de ce Tf $(t)$ cons. nous fait passer de la réalité sociologique du tourisme à son impact économique. Selon la finalité de l'étude, on usera plus ou moins de l'une ou de l'autre de ces mesures.

Nous avons fait une application a titre d'exemple à l'ile de Madere, voici les chiffres utilisés (bien que datant d'une dizaine $\mathrm{d}^{\top}$ années, la valeur de la méthode implique plus dans cet article que son résultat).

\begin{tabular}{lccc}
\hline Nationalites & $\begin{array}{l}\text { Nuites } \\
\text { en } \\
\text { milliers }\end{array}$ & $\begin{array}{l}\text { Rbm } \\
\text { (milliers } \\
\text { de dollars) }\end{array}$ & N $\times \begin{array}{r}\text { Rbm } \\
10^{6}\end{array}$ \\
\hline Allemands & 405,5 & 10,2 & 4136 \\
Belges & 56,5 & 10,2 & 576 \\
Brésil & 10,1 & 1,7 & 17 \\
Canada & 18,7 & 9,3 & 174 \\
Danois & 92,6 & 11,2 & 1037 \\
Espagnols & 62,4 & 4 & 250 \\
Américains & 55,1 & 10,1 & 556 \\
Finlandais & 49,4 & 7,1 & 351 \\
Français & 158 & 8,9 & 1406 \\
Hollandais & 63,1 & 9,7 & 613 \\
Anglais & 587,6 & 5,7 & 3349 \\
Italiens & 30,6 & 5,7 & 174 \\
Norvegiens & 17,7 & 11,4 & 202 \\
Portugais & 503 & 1,9 & 958 \\
Suédois & 137,3 & 10,9 & 1496 \\
Suisses & 18,1 & 14 & 253 \\
\hline Total & & & $\mathbf{1 5 5} 548$ \\
\hline
\end{tabular}

Tf $(t)$ cons.: $\frac{15548 \times 10^{6}}{365 \times 210^{3} \times 2510^{4}}$

Tf $(t)$ cons:: $=0.085$

Le Tf (t) simple est pour Madère de 4,2. Mais ces deux grandeurs ne sont pas comparables et il importe donc d'utiliser l'une ou l'autre.

\section{Conclusion}

Un trop grand nombre d'études touristiques, confiées souvent à des bureaux d'études peu qualifiés, débutent par des préambules de nature qualitative et souvent, faisant appel à des idées toutes faites, $\mathrm{n}$ introduisent pas vraiment le problème qu'il s'agit d'analyser.

On aura intérêt à aborder tout de suite un point de vue quantitatif et numerique et le taux de fonction touristique, soit simple, soit consolidé, a le mérite de faire tout de suite réfléchir sur des grandeurs numériques beaucoup plus significatives pour l'esprit que tout autre considération. C'est la démarche scientifique par excellence, celle du physicien, qui sait assembler des atomes ou celle de l'astronome qui dénombre les étoiles d'une galaxie et donne leur magnitude. Les sciences exactes ne peuvent échapper à cette démarche et l'économie touristique y est déjà fort bien adaptée. Un gros retard pèse encore dans l'expression sociologique du tourisme et bien entendu ces imperfections des sciences de base se retrouvent dans l'aménagement. Le recours à un outil comme celui que nous avons créé il y a 40 ans, si grossier soit-il, représente le passage d'une phase de connaissances à une autre. Le tourisme sort de sa préhistoire et même si les outils dont il use ne sont encore que des silex plus ou moins éclatếs, ils sont suffisants pour apporter la lumière supplémentaire dont nous aurons de plus en plus besoin pour comprendre ce phénomène. $f$

\section{Notes explicatives}

(1) CHET, Fondation Vasaraly, 9, avenue Marcel Pagnol 13000, Aix-en-Provence, France

(2) Mais on peut concenoir la variation de Tt (t) par rapport a $\mathrm{L}$ ou a $\mathrm{P}$ (dériveses). L'analyse algebrique nous entrainerait hors de notre sulet.

(3) L'blaboration d'un taux de fonction touristique prend place dans la ligne d'une recherche de statistiques detat alors que presque tous les vavaux theoriques sur le tourisme ont été conduits surtout autour de "statistiques de movvemenis" et ont médiocrement avanced dans la connaissance du reel. Or, comme l'écrit A. Piatier IStatistique ot observation efconomique, PUF, Paris, p. 130), les statistiques d' that sont d'un plus grand intérét a l'origine d'une recherche que les statistiques de flux et definissent des "existants" qui constituent la structure teonomique elle-mêtme.

(4) Pierre Defert, La localisation touristique, aspects theoriques et pratiques. Collection AIEST, Vol. 7 , Editions Gurten, 6 Weissenbühlweg. Berne, Suisse. $1967,143 \mathrm{p}$

(5) Les ambinagements et les equipements touristiques, Edition du Moniteur des Travaux publics, Paris. France, avtil 1996, p. 52 